\title{
Clinical relevance of right ventricular diastolic stiffness in pulmonary hypertension
}

\author{
Pia Trip ${ }^{1}$, Silvia Rain ${ }^{1,2}$, M. Louis Handoko ${ }^{2,3}$, Cathelijne van der Bruggen ${ }^{1}$, \\ Harm J. Bogaard ${ }^{1}$, J. Tim Marcus ${ }^{4}$, Anco Boonstra ${ }^{1}$, Nico Westerhof ${ }^{1,2}$, \\ Anton Vonk-Noordegraaf ${ }^{1}$ and Frances S. de Man $^{1,2}$
}

Affiliations: ${ }^{1}$ Dept of Pulmonary Medicine, Institute for Cardiovascular Research, VU University Medical Center, Amsterdam, The Netherlands. ${ }^{2}$ Dept of Physiology, Institute for Cardiovascular Research, VU University Medical Center, Amsterdam, The Netherlands. ${ }^{3}$ Dept of Cardiology, Institute for Cardiovascular Research, VU University Medical Center, Amsterdam, The Netherlands. "Dept of Physics, Institute for Cardiovascular Research, VU University Medical Center, Amsterdam, The Netherlands.

Correspondence: Frances S. de Man, VU University Medical Center, Department of Pulmonary Medicine, De Boelelaan 1117, 1081 HV Amsterdam, The Netherlands. E-mail: fs.demandvumc.nl

ABSTRACT Right ventricular (RV) diastolic stiffness is increased in pulmonary arterial hypertension $(\mathrm{PAH})$ patients. We investigated whether RV diastolic stiffness is associated with clinical progression and assessed the contribution of RV wall thickness to RV systolic and diastolic stiffness.

Using single-beat pressure-volume analyses, we determined RV end-systolic elastance (Ees), arterial elastance $(E \mathrm{a}), \mathrm{RV}$-arterial coupling $\left(E_{\mathrm{es}} / E_{\mathrm{a}}\right)$, and RV end-diastolic elastance (stiffness, Eed) in controls $(n=15)$, baseline PAH patients $(n=63)$ and treated PAH patients (survival $>5$ years $n=22$ and survival $<5$ years $n=23)$.

We observed an association between Eed and clinical progression, with baseline Eed $>0.53 \mathrm{mmHg} \cdot \mathrm{mL}^{-1}$ associated with worse prognosis (age-corrected hazard ratio $0.27, \mathrm{p}=0.02$ ). In treated patients, Eed was higher in patients with survival $<5$ years than in patients with survival $>5$ years $(0.91 \pm 0.50$ versus 0.53 $\left.\pm 0.33 \mathrm{mmHg} \cdot \mathrm{mL}^{-1}, \mathrm{p}<0.01\right)$. Wall-thickness-corrected Eed values in PAH patients with survival $>5$ years were not different from control values $\left(0.76 \pm 0.47\right.$ versus $0.60 \pm 0.41 \mathrm{mmHg} \cdot \mathrm{mL}^{-1}$, respectively, not significant), whereas in patients with survival $<5$ years, values were significantly higher $(1.52$ $\pm 0.91 \mathrm{mmHg} \cdot \mathrm{mL}^{-1}, \mathrm{p}<0.05$ versus controls).

$\mathrm{RV}$ diastolic stiffness is related to clinical progression in both baseline and treated PAH patients. RV diastolic stiffness is explained by the increased wall thickness in patients with $>5$ years survival, but not in those surviving $<5$ years. This suggests that intrinsic myocardial changes play a distinctive role in explaining RV diastolic stiffness at different stages of PAH.

@ERSpublications

Right ventricular diastolic stiffness is related to clinical progression in both baseline and treated PAH patients http://ow.ly/K15me

This article has supplementary material available from www.erj.ersjournals.com

Received: Aug 272014 | Accepted after revision: Feb 172015 | First published online: April 162015

Conflict of interest: Disclosures can be found alongside the online version of this manuscript at erj.ersjournals.com

Copyright @ERS 2015 


\section{Introduction}

In pulmonary arterial hypertension (PAH), pulmonary vascular remodelling leads to a typical fourfold increase in pulmonary artery pressure. The right ventricle (RV) copes with this increased pressure by converting from a low-pressure to a high-pressure pump. As long as this adaptation process is successful, cardiac output and oxygen supply to all organs are assured. Important mechanisms to adapt to an increase in pressure include increased muscle mass and enhanced intrinsic myocyte contractility. Both adaptations have been described in PAH patients and explain the observed increase in RV systolic elastance (Ees; a measure of ventricular contractile function) in $\mathrm{PAH}$ patients [1-6].

However, potential consequences of RV systolic adaptation are increased myocardial stiffness and impaired relaxation. Indeed, we recently showed impaired RV diastolic function in PAH patients [1]. Hypertrophy, fibrosis and stiffening of the RV cardiomyocytes all appeared to contribute to the observed RV diastolic stiffness [1]. However, this analysis was performed in end-stage PAH patients. Therefore, it remains unclear whether RV diastolic impairment already plays a role at earlier stages of the disease, and whether it is associated with clinical progression. Because RV hypertrophy is already present at early stages of the disease, it could be hypothesised that the initial increase in RV diastolic stiffness is explained by the increase in wall thickness. This contrasts with end-stage $\mathrm{PAH}$, in which further increases in diastolic stiffness may relate to intrinsic sarcomere stiffening. Therefore, the aims of this study are: 1) to investigate whether increased RV diastolic stiffness is associated with clinical progression in baseline and treated PAH patients; 2) to assess the contribution of RV wall thickness to RV diastolic stiffness in different disease stages; and 3) to describe the change in RV diastolic stiffness during follow-up.

\section{Methods}

Subjects: control, baseline and treated cases

All patients diagnosed with idiopathic and heritable PAH at the VU University Medical Center (Amsterdam, The Netherlands) between August 10, 1989 and February 25, 2014 ( $\mathrm{n}=267$ ) were evaluated for inclusion in the current study [7]. Part of the patient selection procedure has been described previously [6].

For the assessment of the relationship between diastolic stiffness and clinical progression and RV systolic adaptation, baseline treatment-naïve patients with digitally stored good-quality RV pressure recordings and cardiac magnetic resonance imaging (MRI) performed within 4 weeks of right heart catheterisation were included ( $n=63$ ) (fig. 1). Reasons for excluding patients were: no stored RV pressure curves at the time of diagnosis $(n=48)$ and RV pressure curves that were of poor quality $(n=15)$.

The association between high diastolic stiffness and clinical progression was further assessed in treated patients. In addition, these patients were used to determine 1) the relationship between RV diastolic

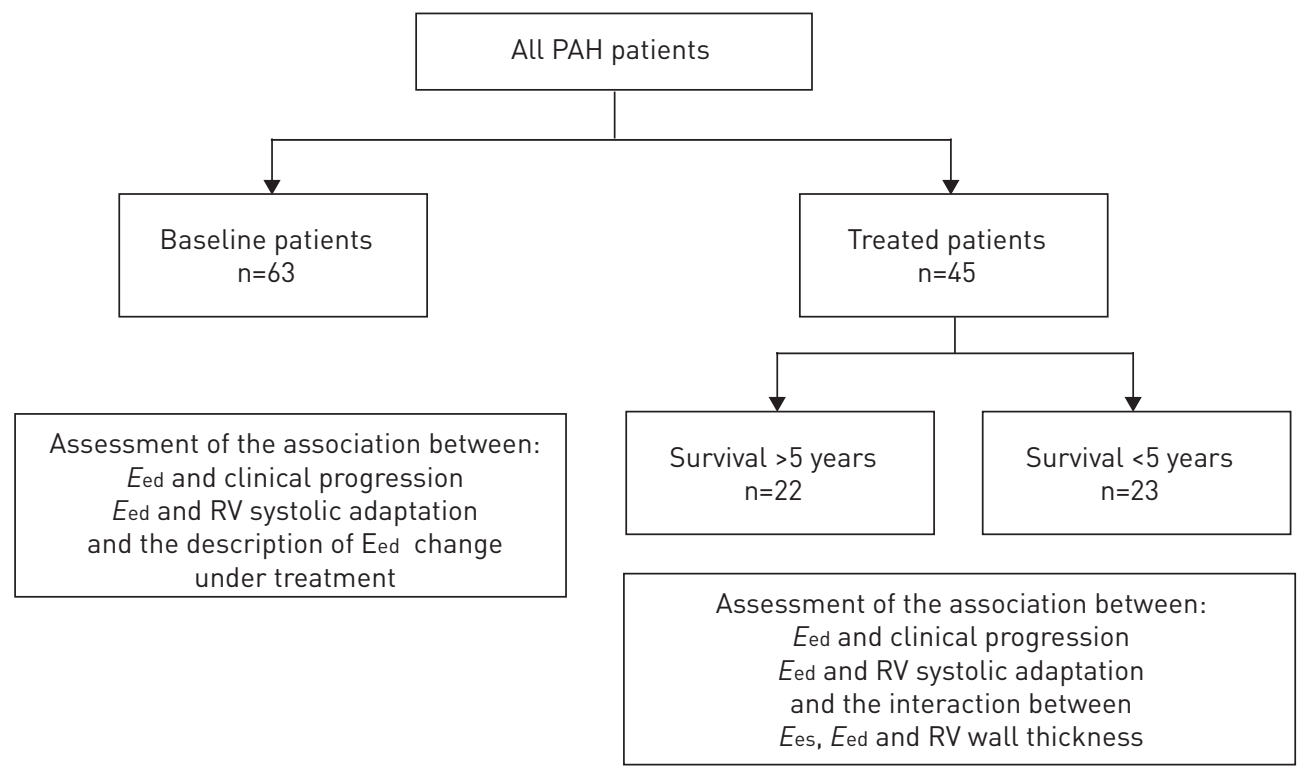

FIGURE 1 Schematic overview of study populations and study aims. PAH: pulmonary arterial hypertension; Eed: end-diastolic elastance; RV: right ventricular; Ees: end-systolic elastance. 
stiffness and RV systolic adaptation and 2) the interaction between RV wall thickness and RV systolic and diastolic function (fig. 1). We retrospectively determined the availability of good-quality RV pressure curves recorded during follow-up and within 4 weeks of cardiac MRI in all idiopathic and heritable PAH patients seen in our hospital. We included patients with these measurements based on survival time after their follow-up assessment. Patients who were alive and who had a follow-up time $<5$ years after the measurement were not included. The remaining patients either survived $>5$ years or died or underwent lung transplantation. These patients were divided into two groups, i.e. survival $<5$ and $>5$ years after the follow-up measurements. Of the latter group, only two out of 22 included patients had an event during follow-up.

In order to assess the change in diastolic stiffness under treatment, we performed a follow-up analysis in a subgroup $(n=30)$ of the baseline patient population in whom RV pressure curves and cardiac MRI were available $0.5-2.5$ years after the baseline measurement.

Subjects referred to the VU University Medical Center between January 1, 2003 and January 1, 2014 for the evaluation of pulmonary hypertension, but who had normal pulmonary artery pressures (mean pulmonary artery pressure (mPAP) $<20 \mathrm{mmHg}$ ) were included as controls if RV pressure recordings with a concomitant cardiac MRI were available $(n=15)$. Due to the retrospective character of this study using data obtained for clinical purposes, the medical ethics review committee of the VU University Medical Center did not consider this study to fall within the scope of the Medical Research Involving Human Subjects Act. Therefore, no additional approval was acquired.

\section{Right heart catheterisation}

Right heart catheterisation was performed as previously described [6]. A detailed description can be found in the online supplementary material. mPAP was averaged over at least two respiratory cycles. Cardiac output was measured by either the direct Fick method or thermodilution. Stroke volume was calculated as cardiac output divided by heart rate. Cardiac output and stroke volume were indexed for body surface area. Total pulmonary vascular resistance (TPVR) was calculated as MPAP and divided by cardiac output.

\section{Cardiac MRI}

All magnetic resonance images were acquired using a 1.5-tesla Avanto or Sonata MRI system equipped with a six-element phased array coil (Siemens Medical Solutions, Erlangen, Germany) as previously described [6]. A stack of short-axis images was taken at breath-hold per slice, with a slice thickness and interslice gap of $5 \mathrm{~mm}$. RV volumes and mass were determined by manually drawing endocardial and epicardial borders at end-diastole and end-systole using mass analysis software (Medis Medical Imaging Systems, Leiden, The Netherlands). End-diastole was defined as the onset of the R-wave of the ECG. End-systole was determined visually as the smallest volume during the cardiac cycle. Relative wall thickness was calculated by dividing RV mass by RV end-diastolic volume. RV ejection fraction (RVEF) = (RVEDV-RVESV)/RVEDV $\times 100 \%$, where RVEDV is RV end-diastolic volume and RVESV is RV end-systolic volume.

\section{Pressure-volume analysis}

Part of the data analysis has been described previously $[1,6,8]$. A detailed description of the data analysis can be found in the online supplementary material. The slope of the end-systolic pressure-volume relationship (Ees) was calculated as follows: $E_{e s}=\left(P_{\text {iso }}-\mathrm{mPAP}\right) /(\mathrm{EDV}-\mathrm{ESV}) . \mathrm{RV}$ isovolumic pressure $\left(P_{\text {iso }}\right)$ per beat was determined according to the single-beat method of SUNAGAWA and co-workers $[9,10]$. Arterial elastance ( $E$ a, a measure of afterload) was calculated by dividing mPAP by stroke volume. RV-arterial coupling (RV systolic adaptation to arterial load) was then calculated as the ratio between Ees and Ea. Diastolic stiffness was assessed by end-diastolic elastance (Eed). The diastolic pressure-volume relationship was determined as described previously (see the online supplementary material) [1]. In previous studies [1], we used $\beta$ (the diastolic stiffness constant) from the exponential pressure-volume relationship described by the formula $P=\alpha\left(e^{\mathrm{V} \beta}-1\right)$ (fig. $\left.2 \mathrm{a}\right)$. Since $\beta$ describes only part of the end-diastolic pressurevolume relationship, we used Eed in the present study. Eed was calculated as the slope of the diastolic pressure-volume relationship at end-diastole using both $\alpha$ and $\beta$ in the following formula: $\alpha \cdot \beta \cdot e^{(\beta \cdot E D V)}$ (fig. $2 a)$. Figure $2 \mathrm{~b}$ shows that Eed and $\beta$ are related ( $\mathrm{r} 0.84, \mathrm{p}<0.001$ ).

\section{Statistical analysis}

The data are presented as mean \pm SEM, unless otherwise stated. A p-value $<0.05$ was considered significant. Survival was calculated from the time of diagnosis to death (all-cause mortality) or lung transplantation. Follow-up was continued until March 1, 2014. A Kaplan-Meier analysis was performed for dichotomised Eed, based on the median and receiver operating characteristic (ROC) derived cut-off values (online fig. $\mathrm{S} 1$ ). The association between these parameters and survival was further explored by Cox regression 

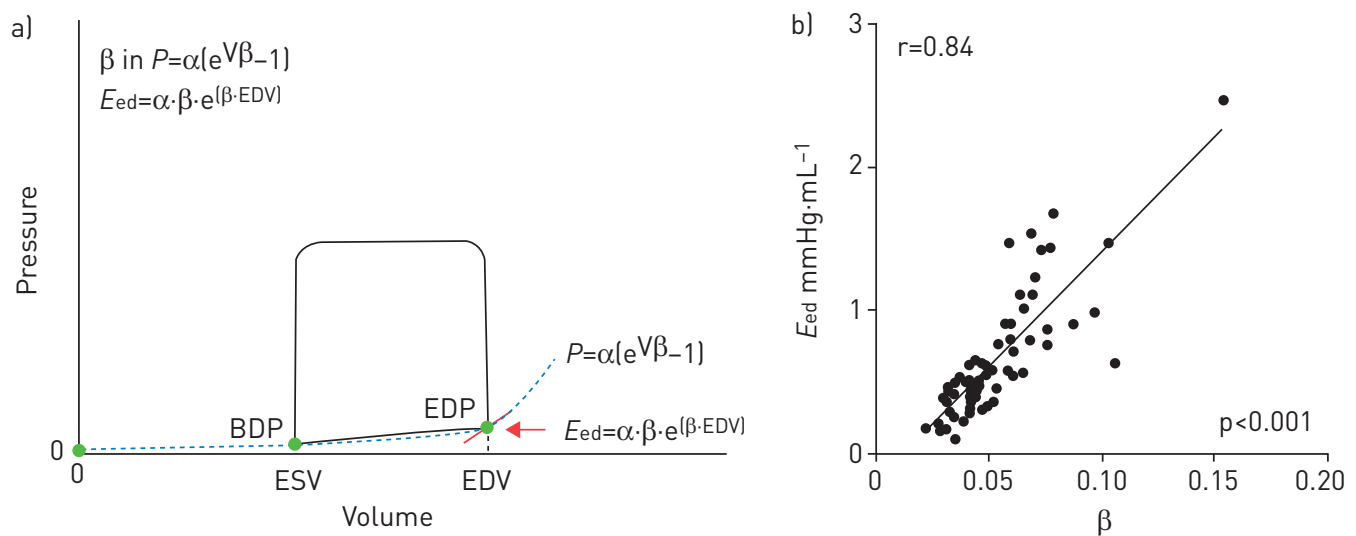

FIGURE 2 a) Two methods to calculate diastolic stiffness. $\beta$ has been used in previous studies [1] and describes only part of the end-diastolic pressure-volume relationship. Eed (end-diastolic elastance) is used in the present study and describes the slope of the end-diastolic pressure-volume relationship at end-diastole. b) Correlation between the previously used measure of diastolic stiffness $\beta$ and Eed in baseline pulmonary arterial hypertension patients. EDV: end-diastolic volume; ESV: end-systolic volume; BDP: begin-diastolic pressure; EDP: end-diastolic pressure.

analysis with correction for age differences. In addition, a univariate Cox regression analysis was used to assess the association between continuous RV systolic and diastolic indices and outcome. Patient characteristics of baseline patients divided into high and low Eed were tested using an independent t-test or Mann-Whitney test, depending on normal distribution. A Chi-squared test was used to compare categorical variables. A one-way ANOVA using Bonferroni's multiple comparison test or a Kruskal-Wallis test with Dunn's multiple comparison was performed, depending on normal distribution, to compare controls and treated PAH patient groups. A linear regression analysis was used to assess the correlation between Eed and Ees. Treatment response was assessed using a paired t-test or a Wilcoxon signed-rank test.

\section{Results}

Relationship between RV diastolic stiffness and clinical progression

To investigate whether RV diastolic stiffness is related to clinical progression, we first compared the clinical characteristics of baseline patients having low and high RV diastolic stiffness (median-based cut-off value $0.53 \mathrm{mmHg} \cdot \mathrm{mL}^{-1}$ ). Table 1 shows that patients with a high diastolic stiffness had a higher mPAP, TPVR and mean right atrial pressure (mRAP) when compared to patients with low diastolic stiffness. RV volumes were similar in the two groups; patients with a high diastolic stiffness exhibited a lower RV ejection fraction and higher RV mass and relative wall thickness. Interestingly, pressure-volume analysis revealed that despite the higher afterload seen in patients with a higher diastolic stiffness, RV-arterial coupling was similar. A survival analysis based on baseline values of RV diastolic stiffness showed that age-corrected survival was worse for patients with a high diastolic stiffness (fig. 3). Similar findings were observed when using an ROC analysis-based optimal Eed cut-off value as presented in the online supplementary material (fig. S1). Univariate Cox regression analysis on RV function indices using continuous variables revealed that higher Eed (hazard ratio (HR) 2.24, 95\% CI 1.05-4.80; p=0.037) and higher $\beta$ (HR 4.75, 95\% CI 1.43-15.85; $\mathrm{p}=0.011$ ) were associated with worse outcome. Of the RV systolic function indices, a lower RVEF (HR 0.94, 95\% CI 0.90-0.99; p=0.011) and lower Ees/Ea (HR 0.15, 95\% CI 0.03-0.69; $\mathrm{p}=0.015)$ were associated with worse outcome. mRAP, cardiac output and Ees were not significantly associated with outcome in this analysis (mRAP HR 1.07, 95\% CI 1.00-1.16; p=0.056; cardiac output HR 0.64, 95\% CI 0.38-1.07; p=0.090; and Ees HR 1.24, 95\% CI 0.73-2.10; p=0.430).

To further investigate the association between RV diastolic stiffness and clinical progression in PAH patients, we divided treated patients into two groups: 1) survival $>5$ years after a follow-up right heart catheterisation ( $\mathrm{PAH}>5$ years) and 2) death occurring within 5 years of a follow-up right heart catheterisation ( $\mathrm{PAH}<5$ years). Table 2 shows general characteristics, haemodynamics and cardiac MRI measurements of patients with $\mathrm{PAH}>5$ years and $\mathrm{PAH}<5$ years, as well as control subjects. As expected, $\mathrm{PAH}<5$ years had a higher RVEDV and a lower RVEF in comparison to $\mathrm{PAH}>5$ years. No difference was observed between $E_{e}$ and $E_{\mathrm{a}}$ in $\mathrm{PAH}>5$ years and $\mathrm{PAH}<5$ years, although $E$ es, $E_{\mathrm{a}}$ and $E$ ed were all increased in comparison to controls (fig. 4). However, in $\mathrm{PAH}<5$ years, but not in $\mathrm{PAH}>5$ years, the reduced $\mathrm{RV}$-arterial coupling coincided with increased RV diastolic stiffness. Together, these data suggest that RV diastolic stiffening is closely associated with clinical progression in both baseline and treated PAH patients. 
TABLE 1 General characteristics and hemodynamics of the total cohort of baseline idiopathic and heritable pulmonary arterial hypertension (PAH) patients divided by low and high right ventricular (RV) diastolic stiffness

\begin{tabular}{|c|c|c|c|}
\hline & \multirow[t]{2}{*}{ Baseline patients } & \multicolumn{2}{|c|}{ PAH patients } \\
\hline & & Low Eed & High Eed \\
\hline Subjects $\mathrm{n}$ & 63 & 32 & 31 \\
\hline Age years & $56(35-71)$ & $58(40-73)$ & $49(30-67)$ \\
\hline Female & $41(65)$ & $21(66)$ & $20(65)$ \\
\hline BSA $\mathrm{m}^{2}$ & $1.9(1.7-2.1)$ & $1.9(1.7-2.1)$ & $1.9(1.7-2.1)$ \\
\hline Length of follow-up years & $2.7(1.4-4.7)$ & $2.7(1.2-8.5)$ & $2.4(1.5-3.9)$ \\
\hline Events & $16(25)$ & $5(16)$ & $11(36)$ \\
\hline \multicolumn{4}{|l|}{ Haemodynamics } \\
\hline $\mathrm{mPAP} \mathrm{mmHg}$ & $53(46-59)$ & $50(45-54)$ & 56 (47-67)* \\
\hline Cardiac index $\mathrm{L} \cdot \mathrm{min}^{-1} \cdot \mathrm{m}^{-2}$ & $2.3(2.0-2.7)$ & $2.5(2.1-2.9)$ & $2.2(2.0-2.7)$ \\
\hline $\mathrm{HR}$ bpm & $80(71-91)$ & $78(68-87)$ & $80(74-97)$ \\
\hline TPVR $\mathrm{mmHg} \cdot \mathrm{min}^{-1} \cdot \mathrm{L}^{-1}$ & $12.3(8.3-15.2)$ & $11.0(7.6-12.9)$ & $14.5(9.5-19.5)^{*}$ \\
\hline mRAP $\mathrm{mmHg}$ & $6(4-11)$ & $5(4-9)$ & $8(5-11)^{*}$ \\
\hline $\mathrm{SvO}_{2} \%$ & $63(56-68)$ & $67(58-70)$ & $61(55-65)$ \\
\hline \multicolumn{4}{|l|}{ Cardiac MRI } \\
\hline RVEDV $\mathrm{mL}$ & $142(122-175)$ & $149(134-166)$ & $140(117-191)$ \\
\hline RVESV mL & $93(70-128)$ & $91(73-112)$ & $99(66-145)$ \\
\hline RVEF \% & $36(23-45)$ & $39(35-47)$ & $27(18-38)^{*}$ \\
\hline RV mass $\mathrm{g}$ & $98(78-118)$ & 90 (77-113) & $112(81-132)^{*}$ \\
\hline Relative wall thickness $\mathrm{g} \cdot \mathrm{mL}^{-1}$ & $0.65(0.55-0.82)$ & $0.59(0.51-0.70)$ & $0.76(0.57-0.84)^{*}$ \\
\hline \multicolumn{4}{|l|}{ Pressure-volume analysis } \\
\hline Ees $\mathrm{mmHg} \cdot \mathrm{mL}^{-1}$ & $1.35(0.99-1.94)$ & $1.12(0.87-1.49)$ & $1.64(1.18-2.59)^{*}$ \\
\hline Ea $\mathrm{mmHg} \cdot \mathrm{mL}^{-1}$ & $1.14(0.78-1.44)$ & $0.86(0.64-1.20)$ & $1.38(1.05-2.09)^{*}$ \\
\hline Eed $\mathrm{mmHg} \cdot \mathrm{mL}^{-1}$ & $0.52(0.37-0.86)$ & $0.37(0.27-0.45)$ & $0.86(0.62-1.22)^{*}$ \\
\hline$E_{\mathrm{es}} / \mathrm{Ea}_{\mathrm{a}}$ & $1.29(0.99-1.57)$ & $1.32(1.00-1.66)$ & $1.25(0.90-1.56)$ \\
\hline \multicolumn{4}{|c|}{$\begin{array}{l}\text { Data are presented as median (interquartile range) or } \mathrm{n}(\%) \text {, unless otherwise stated. Eed: end-diastolic } \\
\text { elastance; BSA: body surface area; mPAP: mean pulmonary artery pressure; HR: heart rate; TPVR: total } \\
\text { pulmonary vascular resistance; mRAP: mean right atrial pressure; Svo }{ }_{2} \text { : mixed venous oxygen saturation; } \\
\text { MRI: magnetic resonance imaging; RVEDV: right ventricular end-diastolic volume; RVESV: right ventricular } \\
\text { end-systolic volume; RVEF: right ventricular ejection fraction; Ees: end-systolic elastance; Ea: arterial } \\
\text { elastance. *: } \mathrm{p}<0.05 \text { versus PAH low Eed. }\end{array}$} \\
\hline
\end{tabular}

$R V$ relative wall thickness and diastolic stiffness in different disease stages

To investigate whether the increased $E$ es and $E$ ed in $\mathrm{PAH}>5$ years and $\mathrm{PAH}<5$ years are a sole consequence of increased RV wall thickness, we subsequently calculated wall thickness corrected Ees and Eed values. As can be observed in figure 5, Ees values remained increased after correction for RV wall thickness. In contrast, wall thickness-corrected Eed values were normal in $\mathrm{PAH}>5$ years, whereas in $\mathrm{PAH}<5$ years wall thickness-corrected Eed values were significantly increased. These data suggest that diastolic stiffness in

FIGURE 3 Survival of baseline patients according to low $(\leqslant 0.53)$ or high $(>0.53)$ end-diastolic elastance $\left(E\right.$ ed). ${ }^{\#}$ : age-corrected.

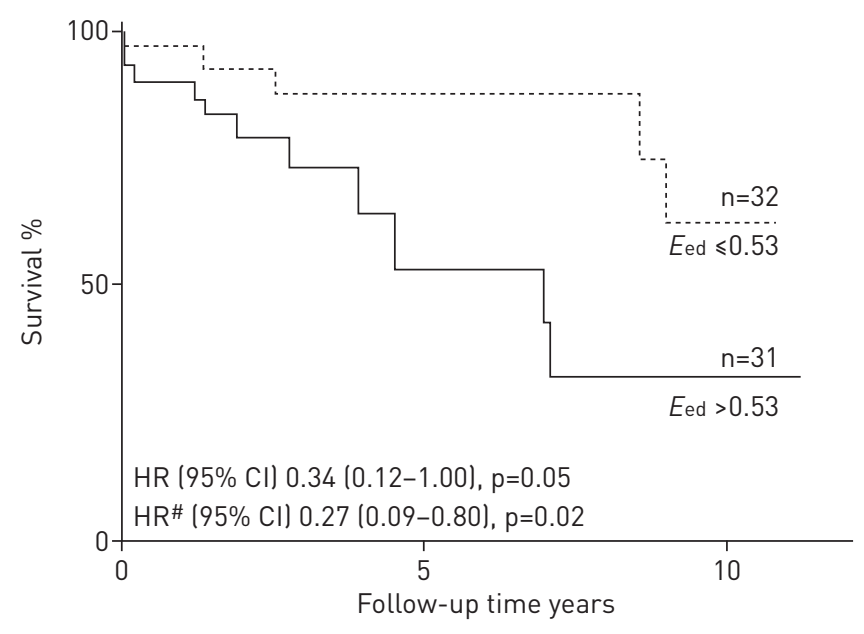


TABLE 2 Clinical characteristics and right ventricular magnetic resonance imaging (MRI) parameters of controls and the cohort of treated idiopathic and heritable pulmonary arterial hypertension (PAH) patients divided based on survival $(<5$ or $>5$ years $)$

\begin{tabular}{|c|c|c|c|}
\hline & \multirow[t]{2}{*}{ Controls } & \multicolumn{2}{|c|}{ PAH patients } \\
\hline & & $\mathbf{P A H}>5$ years & $\mathbf{P A H}<5 y$ ears \\
\hline Subjects $n$ & 15 & 22 & 23 \\
\hline Age years & $49(34-59)$ & $37(31-51)$ & $43(36-54)$ \\
\hline Female n (\%) & 13 (87) & $18(82)$ & $18(78)$ \\
\hline \multicolumn{3}{|l|}{ Haemodynamics } & $1.75(1.62-1.92)$ \\
\hline mPAP $\mathrm{mmHg}$ & $14(12-16)$ & $50(40-61)^{*}$ & $50(43-60) *$ \\
\hline Cardiac index $\mathrm{L} \cdot \mathrm{min}^{-1} \cdot \mathrm{m}^{-2}$ & $3.6(3.1-5.1)$ & $3.1(2.3-3.4)^{*}$ & $2.8(2.2-3.5)^{*}$ \\
\hline $\mathrm{HR}$ bpm & 79 (72-87) & 78 (68-90) & $89(77-100)$ \\
\hline TPVR $\mathrm{mmHg} \cdot \mathrm{min}^{-1} \cdot \mathrm{L}^{-1}$ & $2.1(1.5-2.5)$ & $9.1(6.6-13.8)^{*}$ & $10.2(7.9-14.7) *$ \\
\hline mRAP $\mathrm{mmHg}$ & $4(3-5)$ & $5(2-8)$ & $9(4-13)^{*}$ \\
\hline $\mathrm{SvO}_{2} \%$ & $75(71-80)$ & $67(63-72)^{*}$ & $63(56-69)^{*}$ \\
\hline \multicolumn{4}{|l|}{ Cardiac MRI } \\
\hline RVEDV mL & 120 (94-135) & $138(118-159)$ & $200(136-269)^{*, \#}$ \\
\hline RVESV $\mathrm{mL}$ & $44(37-50)$ & $75(66-114)^{*}$ & $143(92-227)^{*}$ \\
\hline RVEF \% & $63(54-68)$ & $42(33-47)^{*}$ & $22(14-33) * \#$ \\
\hline RV mass g & $37(31-48)$ & $96(81-116)^{*}$ & $125(86-149)^{*}$ \\
\hline Relative wall thickness $\mathrm{g} \cdot \mathrm{mL}^{-1}$ & $0.33(0.27-0.39)$ & $0.70(0.64-0.80)^{*}$ & $0.61(0.47-0.80)^{*}$ \\
\hline \multicolumn{4}{|c|}{$\begin{array}{l}\text { Data are presented as median (interquartile range), unless otherwise stated. BSA: body surface area; } \\
\text { mPAP: mean pulmonary artery pressure; HR: heart rate; TPVR: total pulmonary vascular resistance; } \\
\text { mRAP: mean right atrial pressure; Svo } 0_{2} \text { : mixed venous oxygen saturation; RVEDV: right ventricular } \\
\text { end-diastolic volume; RVESV: right ventricular end-systolic volume; RVEF: right ventricular ejection } \\
\text { fraction; RV: right ventricular. }{ }^{*} \text { : } p<0.05 \text { versus controls; }{ }^{*} \text { : } p<0.05 \text { versus PAH }>5 \text { years. }\end{array}$} \\
\hline
\end{tabular}

stable patients may be largely explained by hypertrophy, while in progressive patients additional intrinsic factors may play a role in increasing diastolic stiffness.

\section{Relationship between RV diastolic stiffness and systolic adaptation}

To assess whether diastolic stiffness is associated with impaired RV systolic adaptation, we investigated the relationship between $E_{\text {ed }}$ and $E$ es $/ E_{\text {a }}$ and between $E_{\text {ed }}$ and $E_{\text {es }}$ in baseline and treated patients. In baseline patients, Eed showed no correlation with $E_{\mathrm{es}} / E_{\mathrm{a}}\left(\mathrm{r}^{2}=0.01, \mathrm{p}=0.53\right)$, but a weak correlation with $E_{\mathrm{es}}\left(\mathrm{r}^{2} 0.25\right.$, $\mathrm{p}<0.001)$. In treated patients, no correlation between Eed and Ees was observed $\left(\mathrm{r}^{2} 0.03, \mathrm{p}=0.264\right)$ and only a weak correlation between Eed and $E$ es/Ea was observed $\left(\mathrm{r}^{2}=0.17, \mathrm{p}=0.005\right)$, suggesting that RV diastolic stiffening and systolic adaptation are largely independent processes.

\section{Change in RV diastolic stiffness under treatment}

To investigate whether diastolic stiffness changes under treatment, we performed a follow-up analysis in a subgroup of the baseline $\mathrm{PAH}$ patients. Online table S1 shows the general characteristics and haemodynamics of the total cohort and of the subgroup at baseline and follow-up. 30 patients were included. Median time between baseline and follow-up was 1.0 year (interquartile range (IQR) $0.8-$ 1.1 years). The subgroup was representative of the total baseline population, as no differences in general characteristics and haemodynamics were observed.

Eed showed a decrease of $>10 \%$ during follow-up in 20 patients. The remaining patients showed an increase in Eed during follow-up. Absolute median change in Eed was $-0.20 \mathrm{mmHg} \cdot \mathrm{mL}^{-1}$ (IQR -0.37$\left.0.12 \mathrm{mmHg} \cdot \mathrm{mL}^{-1}, \mathrm{p}=0.061\right)$. Figure 6 shows individual changes in Eed during follow-up. Also shown are changes in RV relative wall thickness and Eed/relative wall thickness. As can be observed, relative wall thickness decreased ( $\mathrm{p}=0.011)$, while Eed/relative wall thickness did not alter under treatment $(\mathrm{p}=0.175)$.

\section{Discussion}

In the present study we assessed RV diastolic stiffness in a large cohort of baseline and treated PAH patients and demonstrated that: 1) diastolic stiffening is associated with clinical progression in both baseline and treated PAH patients; 2) diastolic stiffness in treated PAH patients with a survival $>5$ years is largely explained by increased RV wall thickness, whereas in PAH patients with a survival $<5$ years RV 

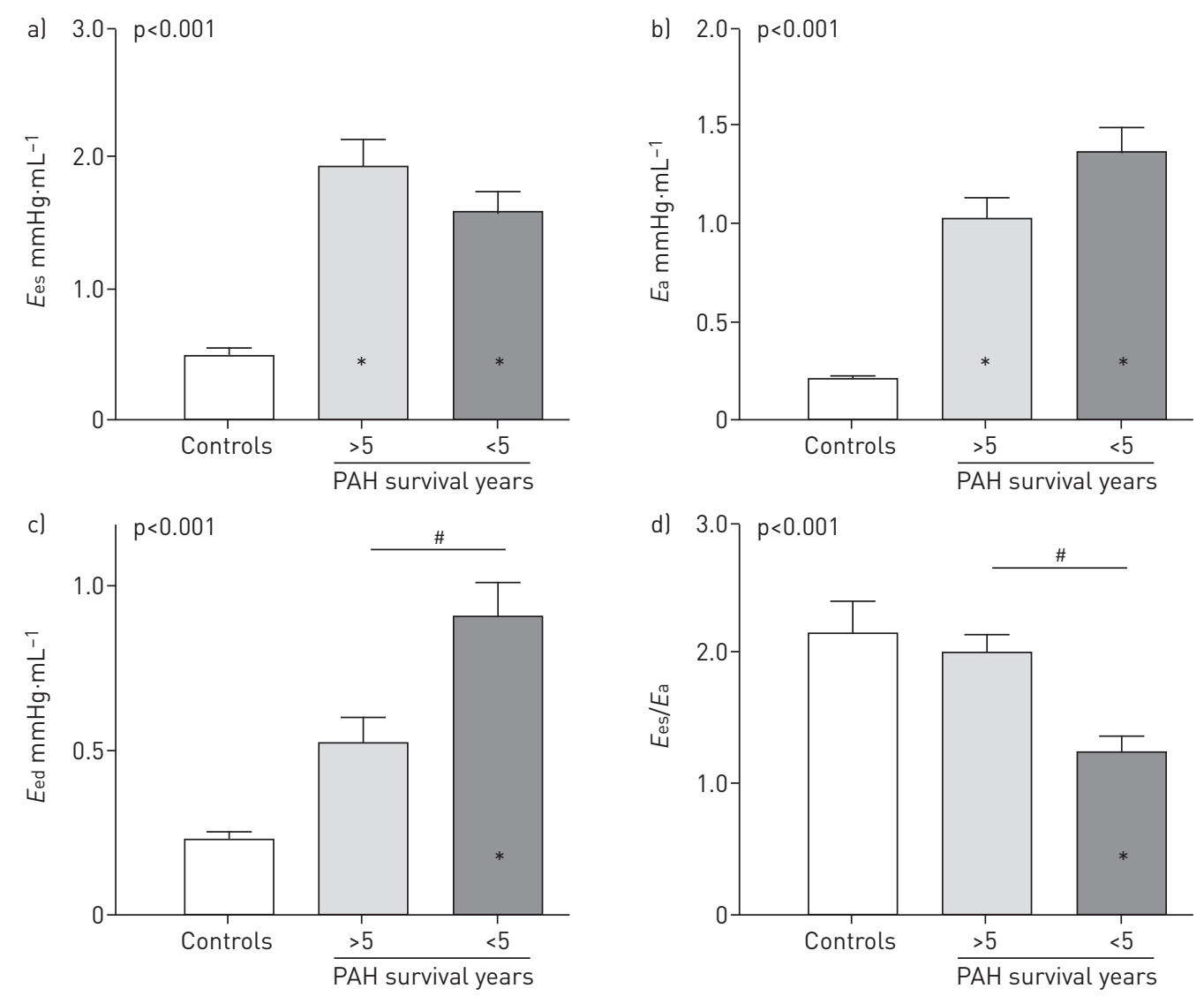

FIGURE 4 Pressure-volume analysis performed in controls $(\mathrm{n}=15)$, pulmonary arterial hypertension (PAH) patients surviving $>5$ years $(\mathrm{PAH}>5$ years) after a follow-up right heart catheterisation $(\mathrm{n}=22)$ and $\mathrm{PAH}$ patients in whom death occurred within 5 years of a follow-up right heart catheterisation $(n=23)$. a) Right ventricular (RV) systolic elastance; b) arterial elastance $\left(E_{\mathrm{a}}\right)$; c) RV diastolic stiffness; d) RV-arterial coupling. Ees: end-systolic elastance; Eed: end-diastolic elastance. ${ }^{*}: \mathrm{p}<0.05$ versus controls; ${ }^{*}: \mathrm{p}<0.05$ versus $\mathrm{PAH}>5$ years.

diastolic stiffness remains increased after correction for RV wall thickness; 3) RV diastolic stiffness is only weakly associated with impaired RV systolic adaptation in treated PAH patients, while no relationship exists in baseline PAH patients; and 4) RV diastolic stiffness decreases $>10 \%$ during follow-up in the majority of $\mathrm{PAH}$ patients, but overall no statistical significant decrease in diastolic stiffness can be observed.
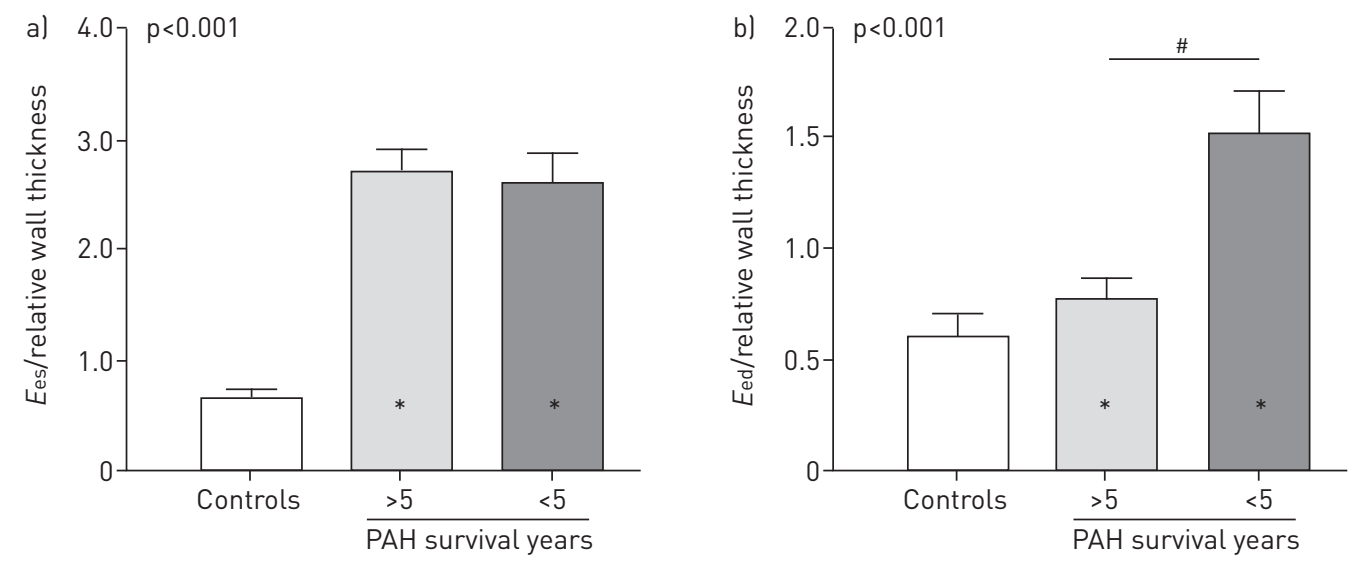

FIGURE 5 Wall-thickness corrected right ventricular a) end-systolic elastance (Ees) and b) end-diastolic elastance (Eed) in controls and treated pulmonary arterial hypertension $(\mathrm{PAH})$ patients surviving $>5$ years $(\mathrm{PAH}>5$ years $)$ and $<5$ years after follow-up right heart catheterisation. ${ }^{*}: \mathrm{p}<0.05$ versus controls; ${ }^{*}: \mathrm{p}<0.05$ versus $\mathrm{PAH}>5$ years. 

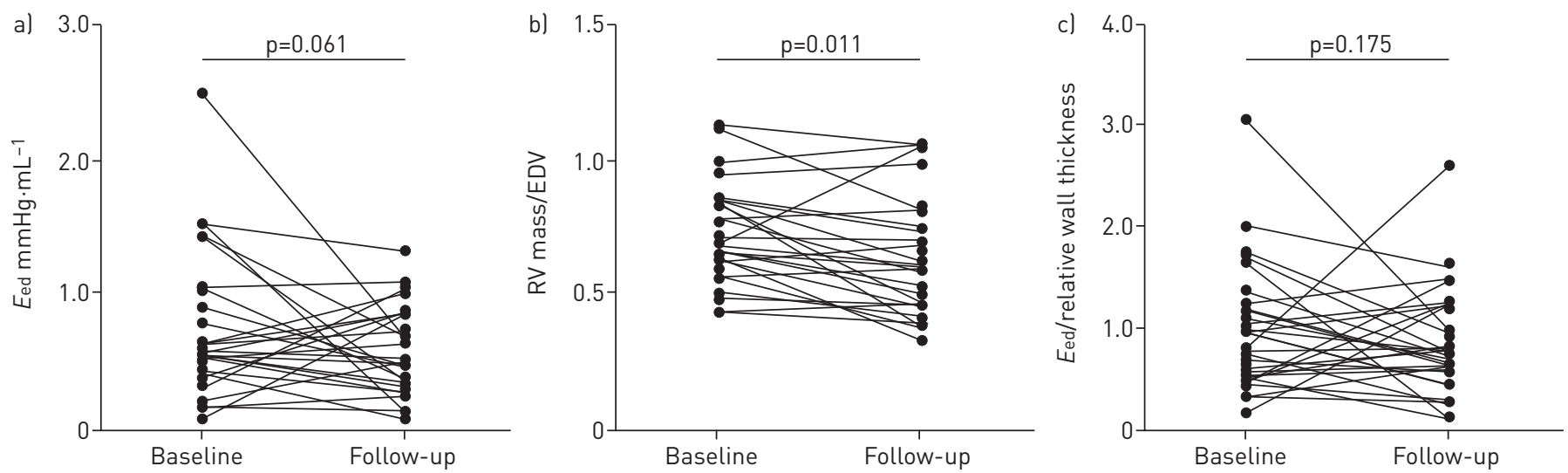

FIGURE 6 Individual changes in right ventricular (RV) a) end-diastolic elastance (Eed); b) relative wall thickness; and c) Eed/relative wall thickness during follow-up. EDV: end-diastolic volume.

\section{The clinical importance of RV diastolic stiffness}

Previous large PAH patient cohort studies have demonstrated the clinical importance of load-dependent measures of RV diastolic stiffening, such as right atrial pressure, increased atrium-dependent RV filling, and prolonged RV isovolumic relaxation [11-15]. In particular, right atrial pressure is one of the parameters that is frequently identified as predictor of survival in multivariate survival analyses [11]. We have recently introduced a novel method to assess RV diastolic stiffness in a load-independent fashion using a single-beat, diastolic pressure-volume analysis [1]. Load-independent assessment of RV diastolic stiffness is scientifically important, as it provides insight into the intrinsic alterations of the RV myocardium independently of the degree of pressure overload. Recently, VANDERPOOL et al. [16] showed that RV diastolic stiffness relates to survival, but this association was not observed when a correction was made for RAP, mPAP and stroke volume. As this analysis was performed in a mixed group of World Health Organization (WHO) group I (including connective-tissue disease-associated PAH) and WHO group II pulmonary hypertension patients, it remains unknown whether load-independent RV diastolic stiffness is related to clinical progression and development of right heart failure in idiopathic PAH patients. Therefore, we investigated the association between clinical progression and RV diastolic stiffness and observed an association in both treated and baseline PAH patients.

Subsequently, we were interested in the relationship between RV diastolic stiffness and RV systolic adaptation. Both systolic and diastolic function are closely modulated on a cellular level by calcium flux and sarcomeric function, and at the ventricular level by wall mass [13, 17]. We identified changes in protein expression of important calcium-handling proteins indicating prolonged diastolic calcium clearance in tissue samples from end-stage PAH patients [18]. Furthermore, increased calcium sensitivity of the sarcomeric proteins may add to RV systolic adaptation, as less calcium would be needed to obtain a similar development of force $[1,18]$. However, at the same time increased calcium sensitivity will affect relaxation of the RV cardiomyocytes, thereby inducing diastolic stiffening of the RV. Therefore, we recently proposed that in early stages of $\mathrm{PAH}, \mathrm{RV}$ diastolic stiffness may result from the adaptation mechanisms that are induced to preserve RV systolic adaptation as long as possible, such as ventricular wall mass and increased calcium sensitivity [19]. In later stages, RV diastolic stiffness will become more prevalent due to stiffening of the RV cardiomyocytes themselves, which will eventually hamper RV systolic adaptation in end-stage PAH $[15,19,20]$. In this study, support for this hypothesis was provided by finding increased RV diastolic stiffness in baseline PAH patients in whom RV systolic adaptation was relatively preserved. Moreover, in treated PAH patients only, RV diastolic stiffness coincided with impaired RV systolic adaptation.

\section{Possible mechanisms of RV diastolic stiffness in PAH}

To obtain further insight into the mechanism of RV diastolic stiffness and systolic function in different stages of PAH, we calculated systolic elastance corrected for relative wall thickness. We used relative wall thickness (EDV/RV mass) as to control for the effect of both volume and hypertrophy on systolic and diastolic stiffness. After correction for wall thickness, RV systolic function was increased in all PAH patients in comparison to controls. This indicates that in addition to the increased RV wall mass, intrinsic alterations in the cardiac muscle are important in determining increased systolic function of the RV in PAH. RV diastolic stiffness corrected for wall thickness was not different from control subjects in PAH patients with a survival $>5$ years. This may indicate that RV diastolic stiffness in early or stable PAH is 
merely a consequence of RV systolic adaptation. In PAH patients with a survival $<5$ years, RV diastolic stiffness is increased out of proportion to the increase in wall thickness, which indicates that intrinsic cardiac muscle alterations play an additional role in determining diastolic stiffness in more advanced PAH. One such intrinsic alteration could be RV cardiomyocyte stiffening due to hypophosphorylation of the giant sarcomeric protein titin, which we recently showed in RV samples of end-stage PAH patients [18]. Another possible mechanism of RV diastolic stiffness may be increased collagen deposition, although until now only modest increases in RV fibrosis have been reported in clinical PAH [1].

\section{Clinical implications}

With the present study we show that in a large baseline and treated PAH patient cohort RV diastolic stiffness is associated with clinical progression. RV diastolic stiffness decreased $>10 \%$ in the majority of patients, but showed no statistical significant decrease in the total group analysis. The minimal decrease in RV diastolic stiffness coincided with a decrease in relative wall thickness, indicating that treatment has little or no effect on intrinsic RV diastolic stiffness. These findings should be subjected to further evaluation and underscore the need to further explore the additive value of the determination of diastolic stiffness in predicting patient outcome. Moreover, future studies are needed to provide tools to noninvasively assess RV diastolic function. Currently, the available noninvasive techniques (echocardiography and MRI-measured relaxation velocities and filling patterns) have significant drawbacks (load dependency) which limit the clinical applicability of noninvasive load-independent RV diastolic evaluation [21-23]. However, studies on the assessment of diastolic wall strain in patients with left ventricular diastolic dysfunction have shown promising results, making diastolic wall strain a possible future evaluation tool for RV diastolic dysfunction in PAH $[24,25]$.

\section{Conclusions}

With the present study we demonstrate that RV diastolic stiffening is associated with clinical progression baseline as well as treated PAH patients. In addition, we show that RV diastolic stiffness in treated PAH patients with a survival $>5$ years is largely explained by increased RV wall thickness, whereas in PAH patients with a survival $<5$ years the further increase in RV diastolic stiffness is most likely related to additional intrinsic alterations of the myocardium. Furthermore, RV diastolic stiffness is only weakly associated with impaired RV systolic adaptation in treated PAH patients, while no relationship exists in baseline PAH patients.

\section{References}

1 Rain S, Handoko ML, Trip P, et al. Right ventricular diastolic impairment in patients with pulmonary arterial hypertension. Circulation 2013; 128: 2016-2025.

2 de Man FS, Handoko ML, van Ballegoij JJ, et al. Bisoprolol delays progression towards right heart failure in experimental pulmonary hypertension. Circ Heart Fail 2012; 5: 97-105.

3 de Man FS, Tu L, Handoko ML, et al. Dysregulated renin-angiotensin-aldosterone system contributes to pulmonary arterial hypertension. Am J Respir Crit Care Med 2012; 186: 780-789.

4 Kuehne T, Yilmaz S, Steendijk P, et al. Magnetic resonance imaging analysis of right ventricular pressure-volume loops: in vivo validation and clinical application in patients with pulmonary hypertension. Circulation 2004; 110: 2010-2016.

5 Tedford RJ, Mudd JO, Girgis RE, et al. Right ventricular dysfunction in systemic sclerosis-associated pulmonary arterial hypertension. Circ Heart Fail 2013; 6: 953-963.

6 Trip P, Kind T, van de Veerdonk MC, et al. Accurate assessment of load-independent right ventricular systolic function in patients with pulmonary hypertension. J Heart Lung Transplant 2013; 32: 50-55.

7 Galiè N, Hoeper MM, Humbert M, et al. Guidelines for the diagnosis and treatment of pulmonary hypertension: the Task Force for the Diagnosis and Treatment of Pulmonary Hypertension of the European Society of Cardiology (ESC) and the European Respiratory Society (ERS), endorsed by the International Society of Heart and Lung Transplantation (ISHLT). Eur Heart J 2009; 30: 2493-2537.

8 Vonk-Noordegraaf A, Westerhof N. Describing right ventricular function. Eur Respir J 2013; 41: 1419-1423.

9 Brimioulle S, Wauthy P, Ewalenko P, et al. Single-beat estimation of right ventricular end-systolic pressure-volume relationship. Am J Physiol Heart Circ Physiol 2003; 284: H1625-H1630.

10 Sunagawa K, Yamada A, Senda Y, et al. Estimation of the hydromotive source pressure from ejecting beats of the left ventricle. IEEE Trans Biomed Eng 1980; 27: 299-305.

11 Benza RL, Gomberg-Maitland M, Miller DP, et al. The REVEAL Registry risk score calculator in patients newly diagnosed with pulmonary arterial hypertension. Chest 2012; 141: 354-362.

12 D'Alonzo GE, Barst RJ, Ayres SM, et al. Survival in patients with primary pulmonary hypertension. Results from a national prospective registry. Ann Intern Med 1991; 115: 343-349.

13 Gan CT, Holverda S, Marcus JT, et al. Right ventricular diastolic dysfunction and the acute effects of sildenafil in pulmonary hypertension patients. Chest 2007; 132: 11-17.

14 Vonk Noordegraaf A, Galiè N. The role of the right ventricle in pulmonary arterial hypertension. Eur Respir Rev 2011; 20: 243-253.

15 Vonk-Noordegraaf A, Haddad F, Chin KM, et al. Right heart adaptation to pulmonary arterial hypertension: physiology and pathobiology. J Am Coll Cardiol 2013; 62: Suppl. 25, D22-D33.

16 Vanderpool RR, Pinsky MR, Naeije R, et al. RV-pulmonary arterial coupling predicts outcome in patients referred for pulmonary hypertension. Heart 2015; 101: 37-43. 
17 Eichhorn EJ, Willard JE, Alvarez L, et al. Are contraction and relaxation coupled in patients with and without congestive heart failure? Circulation 1992; 85: 2132-2139.

18 Rain S, Bos Dda S, Handoko ML, et al. Protein changes contributing to right ventricular cardiomyocyte diastolic dysfunction in pulmonary arterial hypertension. J Am Heart Assoc 2014; 3: e000716.

19 Rain S, Handoko ML, Vonk Noordegraaf A, et al. Pressure-overload-induced right heart failure. Pflügers Arch 2014; 466: 1055-1063.

20 Voelkel NF, Quaife RA, Leinwand LA, et al. Right ventricular function and failure: report of a National Heart, Lung, and Blood Institute working group on cellular and molecular mechanisms of right heart failure. Circulation 2006; 114: 1883-1891.

21 Leong DP, De Pasquale CG, Selvanayagam JB. Heart failure with normal ejection fraction: the complementary roles of echocardiography and CMR imaging. JACC Cardiovasc Imaging 2010; 3: 409-420.

22 Maurer MS, Spevack D, Burkhoff D, et al. Diastolic dysfunction: can it be diagnosed by Doppler echocardiography? J Am Coll Cardiol 2004; 44: 1543-1549.

23 Paulus WJ, Tschöpe C, Sanderson JE, et al. How to diagnose diastolic heart failure: a consensus statement on the diagnosis of heart failure with normal left ventricular ejection fraction by the Heart Failure and Echocardiography Associations of the European Society of Cardiology. Eur Heart J 2007; 28: 2539-2550.

24 Ohtani T, Mohammed SF, Yamamoto K, et al. Diastolic stiffness as assessed by diastolic wall strain is associated with adverse remodelling and poor outcomes in heart failure with preserved ejection fraction. Eur Heart J 2012; 33: $1742-1749$.

25 Takeda Y, Sakata Y, Higashimori M, et al. Noninvasive assessment of wall distensibility with the evaluation of diastolic epicardial movement. J Card Fail 2009; 15: 68-77. 